\title{
The bound-bound and free-free radiative losses for the nonthermal distributions in solar and stellar coronae ${ }^{\star}$
}

\author{
J. Dudík ${ }^{1,2}$, E. Dzifčáková ${ }^{2}$, M. Karlický ${ }^{2}$, and A. Kulinová ${ }^{1,2}$ \\ 1 Department of Astronomy, Physics of the Earth and Meteorology, Faculty of Mathematics, Physics and Informatics, \\ Comenius University, Mlynská Dolina F2, 84248 Bratislava, Slovak Republic \\ e-mail: dudik@fmph.uniba.sk \\ 2 Astronomical Institute of the Academy of Sciences of the Czech Republic, Fričova 298, 25165 Ondřejov, Czech Republic
}

Received 15 December 2010 / Accepted 22 February 2011

\begin{abstract}
Context. The radiative-loss function is an important ingredient in the physics of the solar corona, transition region, and flares. Aims. We investigate the radiative losses due to the bound-bound transitions and bremsstrahlung for nonthermal $\kappa$ - and $n$-distributions. Methods. The bound-bound radiative losses are computed by integrating synthetic spectra. An analytical expression is derived for nonthermal bremsstrahlung. The bremsstrahlung is computed numerically using accurate values of the free-free Gaunt factor.

Results. We find that the changes in radiative-loss functions due to nonthermal distributions are several times greater than the errors due to the missing contribution of the free-bound continuum or errors in atomic data. For $\kappa$-distributions, the radiative-loss functions are in general weaker than for Maxwellian distribution, with a few exceptions caused by the behavior of Fe. The peaks of the radiativeloss functions are in general flatter. The situation is opposite for $n$-distributions, for which the radiative-loss functions have higher and narrower peaks. Local minima and maxima of the radiative-loss functions may also be shifted. The contribution from bremsstrahlung only changes by a few percent except in the extreme nonthermal case of $\kappa=2$. Stability analysis reveals that the X-ray loops are stable against the radiatively-driven thermal instability.
\end{abstract}

Key words. atomic processes - radiation mechanisms: non-thermal - Sun: corona - Sun: X-rays, gamma rays - instabilities

\section{Introduction}

A wide variety of astrophysical models assume that the distribution of electron energies is Maxwellian. This comes from the assumption that the modeled plasma is collisionally dominated. However, it has been shown that the assumption of Maxwellian distribution may not be accurate if the system involves long-range or long-term interactions, i.e., is nonextensive (e.g., Collier 2004; Leubner 2004, 2005, 2008).

In solar and possibly stellar physics, departures from Maxwellian distribution can occur at low plasma densities in combination with strong temperature or density gradients (e.g., Scudder \& Olbert 1979a,b; Roussel-Dupré 1980; Shoub 1983; Owocki \& Scudder 1983; Dufton et al. 1984; Ljepojevic \& MacNiece 1988; Scudder 1992; Pinfield et al. 1999), in the impulsive phase of solar flares (Seely et al. 1987; Dzifčáková et al. 2008; Dzifčáková \& Kulinová 2010; Kulinová et al. 2011) or in situations, where the the average particle energies are not held fixed, but can change by about an order of magnitude (Collier 2004), e.g., due to dynamic coronal heating.

In this paper we deal with two basic types of nonthermal distributions, each named and characterized by a parameter describing the degree of departure from the Maxwellian distribution. The first type of these distributions are $\kappa$-distributions, characterized by a high-energy power-law tail (Vasyliunas 1968). The $\kappa$-distributions are commonly observed in the solar wind,

* The calculated radiative losses for nonthermal distributions are available as two electronic tables at the CDS via anonymous ftp to cdsarc.u-strasbg.fr (130.79.128.5) or via

http://cdsarc.u-strasbg.fr/viz-bin/qcat?]/A+A/529/A103 where they can explain the observed $\mathrm{O}$ vII/O viII ratios (Owocki $\&$ Scudder 1983) and yield a good fit to the observed electron velocity distribution with $\kappa \approx 4$ (Maksimovic et al. 1997) or lower ( $\kappa=2.6$, Zouganelis 2008). Collier et al. (1996) conclude that the observed velocity distribution of ions in the solar wind is also well-fitted by the $\kappa$-distribution.

Vocks \& Mann (2003) show that the nonthermal tails in the solar wind can originate in the solar corona. Pinfield et al. (1999) conclude that nonthermal distributions with high-energy tails may explain observed Si III line ratios in the transition region spectra observed by SOHO/SUMER (Wilhelm et al. 1995). Dzifčáková \& Kulinová (2011) show that the observed Si III line ratios are indeed explained well with $\kappa$-distributions, once the effect of photoexcitation is taken into account. Using their diagnostic technique, they find that $\kappa=7$ in the active region, $\kappa \approx 11$ in the quiet Sun region, and $\kappa \approx 13$ for the coronal hole. The diagnostic method also works for non-isothermal plasma characterized by differential emission measure (DEM). Diagnostic methods for $\kappa$-distributions using the $\mathrm{Fe}$ lines observed by extreme-ultraviolet spectrometers EIS (Culhane et al. 2007) onboard the HINODE spacecraft (Kosugi et al. 2007) or CORONAS-F/SPIRIT instrument (Zhitnik et al. 2005) were developed by Dzifčáková \& Kulinová (2010). These diagnostic methods are quite complex because the value of $\kappa$ must be diagnosed together with the plasma temperature $T$ and electron density $n_{\mathrm{e}}$. The $\kappa$-distributions of mean electron spectra are consistent with the hard X-ray emission of some coronal sources during partially occulted flares (Kašparová \& Karlický 2009). Formation of the nonthermal distributions in connection to $\kappa$-distributions in solar conditions have been studied by e.g. 


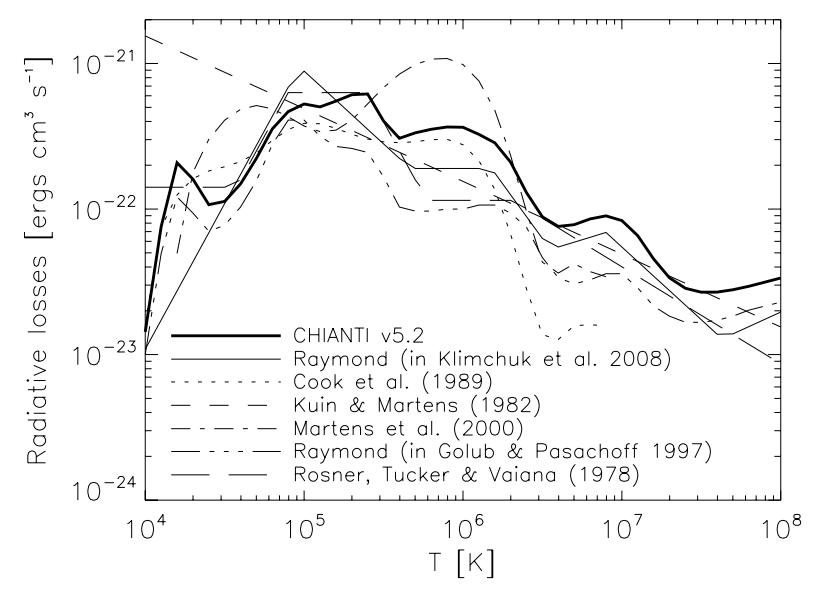

Fig. 1. Radiative-loss functions from the works of Cook et al. (1989), Golub \& Pasachoff (1997), Martens et al. (2000), Klimchuk et al. (2008), and radiative-loss function as given by CHIANTI v5.2 (Landi et al. 2006). Parametric fits by Kuin \& Martens (1982) and Rosner et al. (1978) are also displayed.

Yoon et al. (2006), Rhee et al. (2006), and Vocks et al. (2008). A review of $\kappa$-distributions can be found in Pierrard \& Lazar (2010).

The second type of distributions are the $n$-distributions. Their occurrence during the impulsive phase of flares was first proposed by Seely et al. (1987). Distribution with similar characteristics can form during flares owing to the return current induced by a high-energy electron beam (Dzifčáková \& Karlický 2008). Modeling of the observed Si flare spectra obtained by the RESIK instrument (Sylwester et al. 2005) led Dzifčáková et al. (2008) to conclude that the line intensities of the dielectronic Si xiId lines are reproduced best if the $n$-distributions is assumed. The intensities of the allowed Si XIII and Si XIV line were also reproduced. Kulinová et al. (2011) shows that the diagnostics of the parameter $n$ from both line and continuum spectra yield consistent results. The value of parameter $n$ is time-dependent, with highest detectable values of $n \approx 11$.

The diagnostics of the nonthermal distributions in the transition region and corona during or out of solar flares has potentially far-reaching consequences. This is because emission is the principal source of information about the physical conditions in the emitting plasma and the nonthermal distributions influence both the ionization and excitation equilibrium, i.e., line intensities (e.g., Anderson et al. 1996; Dzifčáková 1992, 1998, 2000, 2002, 2006a; Dzifčáková \& Kulinová 2001, 2003; Wannawichian et al. 2003) and also bremsstrahlung (Kulinová et al. 2011; Tandberg-Hanssen \& Emslie 1988, and references therein). Changes in line intensities due to nonthermal distributions then lead to changes in the filter responses to plasma emission (Dudík et al. 2009a) and can also lead to changes in the total radiative losses from the plasma.

The radiative losses as the function of temperature and electron density have been computed for solar plasma by a number of authors using different sets of atomic data and abundances (e.g., Tucker \& Koren 1971; McWhirter et al. 1975; Cook et al. 1989; Landi \& Landini 1999; Martens et al. 2000; Landi et al. 2006; Colgan et al. 2008; Dere et al. 2009), but all of these works assume the presence of Maxwellian distribution. A sample of them is shown in Fig. 1. The radiative-loss function is a very important ingredient of physical models of solar corona (e.g. Müller et al. 2003; Schrijver et al. 2004; Gudiksen \& Nordlund 2005a,b; Warren \& Winebarger 2006, 2007; Brooks \& Warren 2008;
Klimchuk et al. 2008, 2010; Warren et al. 2010a), which are done to constrain the coronal heating problem (see e.g. Aschwanden 2006; Klimchuk 2006, for a review of the coronal heating problem).

In this paper, we compute the bound-bound and free-free radiative losses for the nonthermal $\kappa$ - and $n$-distributions. The nonthermal distributions are defined in Sect. 2. The computational method is presented in Sect. 3, and the results given in Sect. 4. Section 5 discusses the influence of atomic datasets, electron density, and photoexcitation on the computed radiativeloss functions. It also contains the power-law fits to the radiativeloss functions and a discussion of the thermal stability of coronal loops. The conclusions are summarized in Sect. 6. Piecewise power-law fits to the obtained radiative-loss functions are given in Appendix A.

\section{Nonthermal distributions}

The term "nonthermal electron distribution" refers to any distribution function of electron kinetic energies $E$ that is different from the Maxwellian (thermal) distribution

$f(E) \mathrm{d} E=\frac{2}{\sqrt{\pi}\left(k_{\mathrm{B}} T\right)^{3 / 2}} E^{1 / 2} \mathrm{e}^{-E / k_{\mathrm{B}} T} \mathrm{~d} E$,

where $k_{\mathrm{B}} \approx 1.38 \times 10^{-16} \mathrm{erg} \mathrm{K}^{-1}$ is the Boltzmann constant and $T>0$ represents thermodynamic temperature, which is a parameter of the distribution.

\subsection{The $k$-distributions}

The $\kappa$-distribution is a two-parametric distribution function. It takes the form (e.g., Owocki \& Scudder 1983; Dudík et al. 2009a)

$f(E, \kappa) \mathrm{d} E=A_{\kappa} \frac{2}{\sqrt{\pi}\left(k_{\mathrm{B}} T\right)^{3 / 2}} \frac{E^{1 / 2} \mathrm{~d} E}{\left(1+\frac{E}{(\kappa-3 / 2) k_{\mathrm{B}} T}\right)^{\kappa+1}}$,

where $T$ and $\kappa \in(3 / 2, \infty)$ are the parameters of the distribution. The $A_{\kappa}$ is a normalization constant given by

$A_{\kappa}=\frac{\Gamma(\kappa+1)}{\Gamma(\kappa-1 / 2)(\kappa-3 / 2)^{3 / 2}}$.

The Maxwellian distribution is a special case of $\kappa$-distribution recovered for $\kappa \rightarrow \infty$. The lower limit case of $\kappa \rightarrow 3 / 2$ corresponds to the maximal departures from the Maxwellian distribution. We note that the representation of $T$ as the thermodynamic temperature is valid for the $\kappa$-distributions only in terms of the mean energy $\langle E\rangle$ of the distribution, which is for both $\kappa^{-}$and Maxwellian distributions given by

$\langle E\rangle=\frac{3}{2} k_{\mathrm{B}} T$.

The Maxwellian distribution and the $\kappa$-distributions with the value of $\kappa=2,3,5$, and 10 are for $T=10^{6}$ and $10^{7} \mathrm{~K}$ plotted in Fig. 2. On one hand, $\kappa$-distributions exhibit an enhanced number of particles with both low and high energies. On the other, the number of particles with the intermediate kinetic energies corresponding to the peak of the Maxwellian distribution is less than for the Maxwellian distribution. This is important since the highenergy tail influences the ionization balance of ions with relatively high ionization energies, while the low-energy electrons mostly influence recombination. Thus, both of the two competing mechanisms influencing the ionization equilibrium have increased rates due to the $\kappa$-distributions. 
J. Dudík et al.: The bound-bound and free-free radiative losses for the nonthermal distributions in solar and stellar coronae

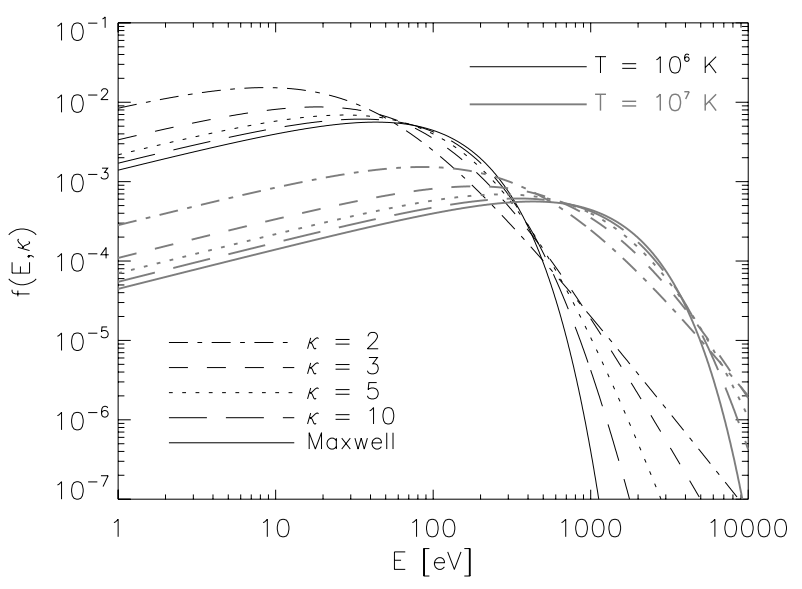

Fig. 2. The $\kappa$-distributions as functions of $E$ and $\kappa$, with $T=10^{6}$ and $10^{7} \mathrm{~K}$, plotted for $\kappa=2,3,5$, and 10 , together with the Maxwellian distribution.

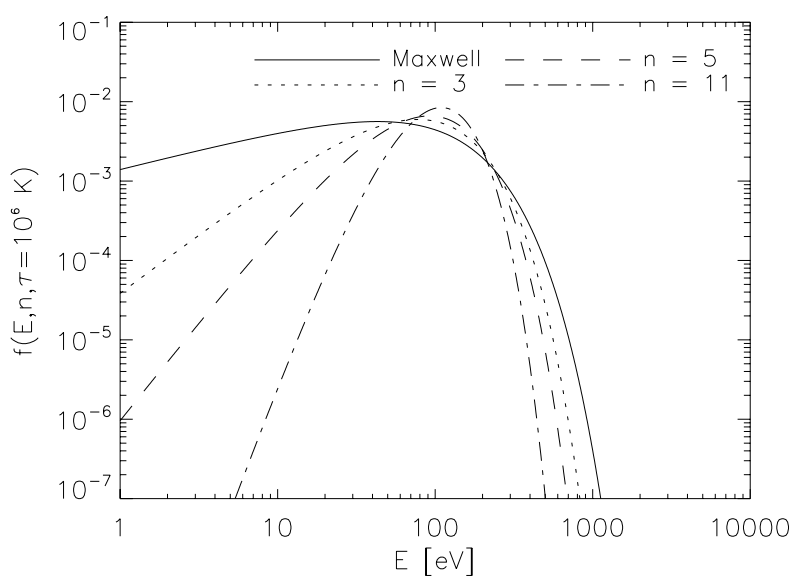

Fig. 3. The $n$-distributions as functions of $E$ and $n$, plotted for $n=1,3$, 5 , and 11 , and $\tau=10^{6} \mathrm{~K}$.

\subsection{The n-distributions}

Another type of nonthermal distribution is the $n$-distribution (e.g., Seely et al. 1987; Dzifčáková \& Tóthová 2007)

$f(E, n) \mathrm{d} E=B_{n} \frac{2}{k_{\mathrm{B}} T \sqrt{\pi}}\left(\frac{E}{k_{\mathrm{B}} T}\right)^{n / 2} \mathrm{e}^{-E / k_{\mathrm{B}} T} \mathrm{~d} E$,

where $B_{n}$ is the normalization constant given by

$B_{n}=\frac{\sqrt{\pi}}{2 \Gamma(n / 2+1)}$

and $n \in\langle 1, \infty)$ is the parameter of the distribution. For $n=1$, the distribution is Maxwellian.

The mean energy of the $n$-distributions depends on $n$

$\langle E\rangle=\frac{3}{2} k_{\mathrm{B}} \tau=\left(\frac{n}{2}+1\right) k_{\mathrm{B}} T$,

where $\tau$ is pseudo-temperature (Dzifčáková 2000) and $T$ is just a parameter of the distribution. The $n$-distributions with the same $\tau$ (i.e., mean energy) are plotted in Fig. 3 . The $n$-distributions with the same $\tau$ have their peaks higher and narrower than to the Maxwellian distribution; i.e., they have decreased number electrons with both high and low energies, but have an increased number of electrons with intermediate energies.

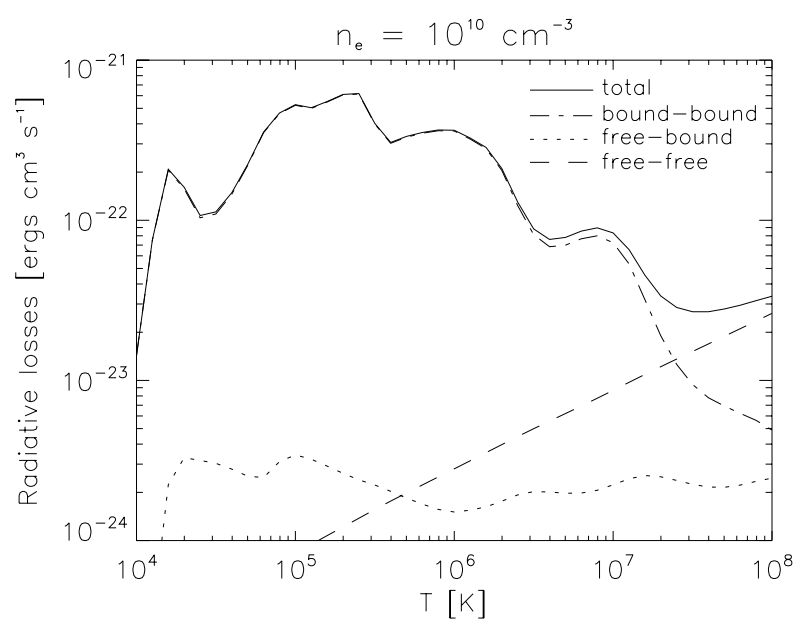

Fig. 4. Contributions to the total radiative losses from the bound-bound, free-bound, and free-free electron transitions for the assumed value of $n_{\mathrm{e}}=10^{10} \mathrm{~cm}^{-3}$, coronal abundances, and Maxwellian distribution.

To keep the notation throughout the paper consistent with the literature, we use $T$ for $\kappa$-distributions and $\tau$ for $n$-distributions as the free parameter describing the mean energy of the distribution.

\section{Computational method}

The total radiative losses $E_{\mathrm{R}}\left[\mathrm{erg} \mathrm{cm}^{-3} \mathrm{~s}^{-1}\right.$ ] per unit volume and unit time from an optically thin plasmas characterized by values of $T$, electron density $n_{\mathrm{e}}$, and the type of distribution are given by the integral of the plasma emission spectrum with the emissivity $\varepsilon$, i.e.,

$E_{\mathrm{R}}=\int_{0}^{\infty} \varepsilon\left(\lambda, A, T, n_{\mathrm{e}}\right.$, distribution $) \mathrm{d} \lambda$,

where $\lambda$ represents the wavelength and $A$ the chemical composition (elemental abundances) of the emitting plasma. The emissivity contains contributions from emission lines, i.e. the boundbound transitions, and also continuum, i.e. the free-bound, and free-free electron transitions. The contribution of bound-bound, free-bound and free-free radiative losses to the total radiative losses for Maxwellian distribution, calculated with CHIANTI v5.2 is depicted in Fig. 4. This figure is similar to Fig. 1 of Landi \& Landini (1999), but with decoupled contributions from the free-bound and free-free transitions.

\subsection{The bound-bound radiative losses}

The emissivity $\varepsilon_{i j}$ of a spectral line with wavelength $\lambda_{i j}$ corresponding to the transition $i \rightarrow j, i>j$, in a $k$-times ionized ion of the element $x$, is given by

$$
\begin{aligned}
\varepsilon_{i j} & =\frac{h c}{\lambda_{i j}} A_{i j} n_{i}=\frac{h c}{\lambda_{i j}} \frac{A_{i j}}{n_{\mathrm{e}}} \frac{n_{i}}{n_{k}} \frac{n_{k}}{n_{x}} A_{x} n_{\mathrm{e}} n_{\mathrm{H}} \\
& =G_{x, i j}\left(\lambda_{i j}, A_{x}, T, n_{\mathrm{e}}, \text { distribution }\right) n_{\mathrm{e}} n_{\mathrm{H}},
\end{aligned}
$$

where $h \approx 6.62 \times 10^{-27}$ erg is the Planck constant, $c \approx 3 \times$ $10^{10} \mathrm{~cm} \mathrm{~s}^{-1}$ represents the speed of light, $A_{i j}$ the Einstein coefficient of spontaneous emission, $n_{i}$ is the density of the ion $k$ with electron on the excited level $i, n_{k}$ the total density of ion $k, n_{x}$ 
the density of element $x$ whose abundance is $A_{x}$, and $n_{\mathrm{H}}$ the hydrogen density. In the coronal approximation (e.g., Aschwanden 2006, p. 58), the ratio $n_{k} / n_{x}$ is given by the ionization equilibrium making it a function of $T$ or $\tau$ and of an assumed type of distribution, while the ratio $n_{i} / n_{k}$ is given by the excitation equilibrium. Since the excitation processes are dominantly collisional, this ratio is a function of electron density $n_{\mathrm{e}}$, type of the electron distribution, and $T$ or $\tau$. The $G_{x, i j}$ denotes the contribution function of the particular emission line.

The total radiative losses per unit volume and unit time due to bound-bound transitions are thus for the assumed type of distribution simply given by the sum

$E_{\mathrm{R}, \mathrm{bb}}=\int_{0}^{\infty} \sum_{x} \sum_{i>j} G_{x, i j}\left(\lambda_{i j}, A_{x}, T, n_{\mathrm{e}}\right.$, distribution $) n_{\mathrm{e}} n_{\mathrm{H}} \mathrm{d} \lambda$.

\subsection{Nonthermal bremsstrahlung}

The bremsstrahlung is the dominant contributor to free-free continuum in solar coronal conditions. The emissivity $\varepsilon_{x, k}$ of bremsstrahlung arising from the interactions between free electrons with the initial energy $E$ and ions of element $x$ in $k$-th stage of ionization is given by (e.g., Phillips et al. 2008, Eq. (4.111) therein)

$\varepsilon_{x, k}=\frac{32 \pi^{2}}{3 \sqrt{3}} \frac{k^{2} e^{6}}{m_{\mathrm{e}}^{2} c^{3}} \sqrt{\frac{m_{\mathrm{e}}}{2 E}} \frac{n_{k}}{n_{x}} A_{x} n_{\mathrm{e}} n_{\mathrm{H}} g_{\mathrm{ff}}(E, \lambda)$,

where $e \approx 4.8 \times 10^{-10}$ statcoulomb is the elemental charge, $m_{\mathrm{e}} \approx$ $9.1 \times 10^{-28} \mathrm{~g}$ is the electron mass, and $g_{\mathrm{ff}}(E, \lambda)$ is the free-free Gaunt factor.

The total bremsstrahlung radiative losses are obtained by summing through all ions of all elements and integrating Eq. (11) over wavelength $\lambda$ and distribution of electron energies. For $\kappa$ - and $n$-distributions, the total bremsstrahlung radiative losses are

$E_{\mathrm{R}, \mathrm{ff}}(T, \kappa)=A_{\kappa} C T^{1 / 2} \int_{0}^{\infty} \int_{0}^{\infty} \frac{g_{\mathrm{ff}}(y, z)}{\left(1+\frac{y+z}{\kappa-3 / 2}\right)^{\kappa+1}} \mathrm{~d} y \mathrm{~d} z$

and

$E_{\mathrm{R}, \mathrm{ff}}(T, n)=B_{n} C T^{1 / 2} \int_{0}^{\infty} \int_{0}^{\infty} g_{\mathrm{ff}}(y, z)(y+z)^{\frac{n-1}{2}} \mathrm{e}^{-y-z} \mathrm{~d} y \mathrm{~d} z$,

respectively. The value of $C$ depends on the assumed chemical composition, ionization balance, and electron density

$C=\frac{32 \pi}{3} \frac{e^{6}}{m_{\mathrm{e}} c^{3} h} \sqrt{\frac{2 \pi k_{\mathrm{B}}}{3 m_{\mathrm{e}}}} n_{\mathrm{e}} n_{\mathrm{H}} \sum_{x} \sum_{k} k^{2} \frac{n_{k}}{n_{x}} A_{x}$.

The $y$ and $z$ are dummy variables that arised from the substitutions

$z=\frac{h c}{\lambda k_{\mathrm{B}} T}$,

$y=\frac{E}{k_{\mathrm{B}} T}-\frac{h c}{\lambda k_{\mathrm{B}} T}$.

These substitutions are the same as those used by, e.g., Phillips et al. (2008, p. 102 therein) in derivation of bremsstrahlung emissivity for the Maxwellian distribution.
We note that the influence of the nonthermal distributions on the bremsstrahlung radiative losses are through the weighting of the Gaunt factor (i.e., the integral) and through the ionization balance $n_{k} / n_{x}$. The $T^{1 / 2}$ dependence of bremsstrahlung on temperature for Maxwellian distribution is retained for both $\kappa$ and $n$-distributions. Rewriting expression (13) as a function of $\tau^{1 / 2}$ would require an additional $((n+2) / 3)^{1 / 2}$ factor because of Eq. (7).

The nonthermal bremsstrahlung spectrum as a function of $T$ can be recovered from Eqs. (12) and (13) by dropping the integral with respect to $z$. The dependence of $y$ on $\lambda$ in Eq. (16) is of no consequence, since $y$ is a dummy variable. The X-ray bremsstrahlung spectrum was for $\kappa$-distributions computed by Kašparová \& Karlický (2009).

\subsection{Computation of the synthetic spectra}

The synthetic spectra needed for computing the bound-bound radiative losses in this paper were obtained using the CHIANTI atomic database and software package, version 5.2 (Dere et al. 1997; Landi et al. 2006). Among other things, CHIANTI contains ionization equilibrium files, procedures for computation of the excitation equilibrium and finally the spectral synthesis.

However, the data and procedures included in CHIANTI all assume the Maxwellian distribution. For the nonthermal $\kappa$ - and $n$-distributions, we used the modification of the CHIANTI developed by Dzifčáková (2006b). This modification includes the ionization equilibrium data files for various integer values of $\kappa$ and $n$ obtained from the works of Dzifčáková (1998, 2002), Dzifčáková \& Kulinová (2003), and Dzifčáková (2006b). Up to this date, the ionization equilibrium files only include data for twelve elements - C, N, O, Ne, Mg, Al, Si, S, Ar, Ca, Fe, and $\mathrm{Ni}$. Ionization equilibria for other elements and the contribution of the free-bound continua have not yet been investigated.

We note that, under coronal conditions, the ionization equilibrium is only governed by processes of direct electron ionization, autoionization, and radiative and dielectronic recombination. The effect of ion-ion collisions on the ionization equilibrium is negligible. The ionization equilibrium thus depends only on the electron distribution function.

The modification of CHIANTI include procedures for computation of emission line spectra for nonthermal distributions as well. The computation of excitation equilibrium involves the processes of electron collisional excitation and deexcitation, spontaneous and stimulated emission, photoexcitation, and proton collisional excitation. The effect of proton collisional excitation is much less than electron excitation. Because of this, we assume that the effect of proton excitation is independent of the actual proton distribution function. Thus, the effect of proton excitation is computed as the proton excitation for Maxwellian distribution with the mean energy equal to the mean energy of the nonthermal electron distribution.

The nonthermal bremsstrahlung spectrum is computed using the approach outlined in Sect. 3.2. In this computation, we use the values of the free-free Gaunt factor $g_{\mathrm{ff}}(E, v)$ obtained by Sutherland (1998). The Maxwellian-averaged values of $\left\langle\bar{g}_{\mathrm{ff}}\right\rangle$ used by the ff_rad_loss.pro procedure of the CHIANTI is also derived in Sutherland (1998).

In this work, we use the coronal elemental abundances that are obtained from the sun_coronal_ext.abund file. These abundances are based on the works of Feldman et al. (1992), Grevesse \& Sauval (1998) and Landi et al. (2002). We utilize the mazzotta_etal_ext.ioneq ionization equilibrium file based on the 
J. Dudík et al.: The bound-bound and free-free radiative losses for the nonthermal distributions in solar and stellar coronae

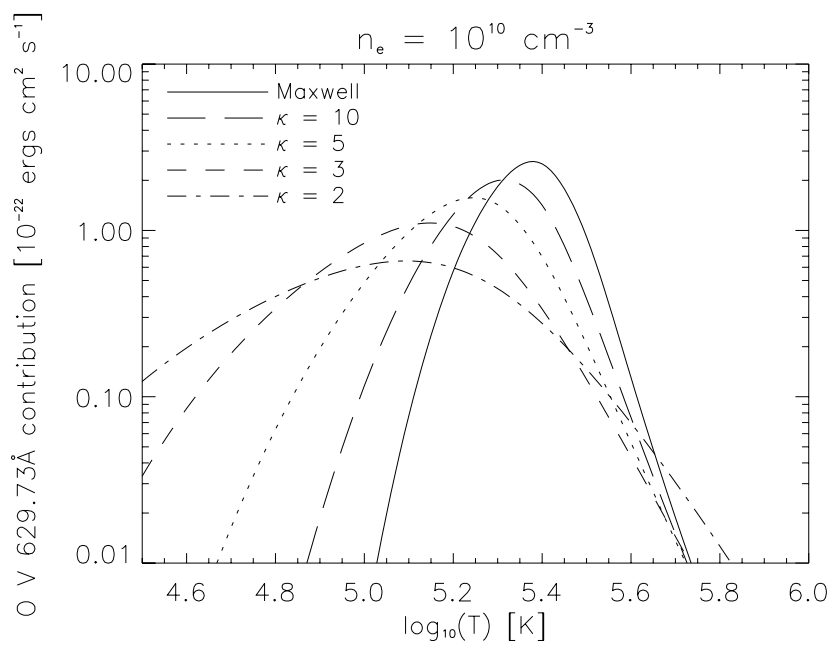

\subsection{Contribution of the free-bound continuum and lines of missing elements for Maxwellian distribution}

The purpose of this section is to evaluate the contribution to total radiative losses arising from the free-bound continuum and emission lines due to the elements with unknown ionization equilibrium for nonthermal distributions, i.e., elements other than $\mathrm{C}, \mathrm{N}, \mathrm{O}, \mathrm{Ne}, \mathrm{Mg}, \mathrm{Al}, \mathrm{Si}, \mathrm{S}, \mathrm{Ar}, \mathrm{Ca}, \mathrm{Fe}$, and Ni. This evaluation is done for the Maxwellian distribution, for which all the neccessary data are known. We note that for Mawellian distribution, the bb_rad_loss.pro, fb_rad_loss.pro and ff_rad_loss.pro routines of CHIANTI allows computation of the contributions from bound-bound, free-bound, and free-free transitions to the total radiative losses directly, i.e., without actual integration of the spectrum. The output of these procedures is displayed in Fig. 4.

The radiative losses from the free-bound continuum are fairly stable for the transition region and for coronal and flare temperatures (Fig. 4). Their relative contribution to the total radiative losses is $\leq 3 \%$ for $\log _{10}(T) \in\langle 4,7\rangle$, with $<1 \%$ for $\log _{10}(T)$ between 5 and 6.1. The contribution rises sharply near the local radiative-loss curve minimum around $\log _{10}(T) \approx 7.5$ to about $8.5 \%$. Since the free-bound transitions only make a small contribution to the radiative losses for the Maxwellian distribution, we do not consider these transitions in the calculation of the radiative losses for the nonthermal distributions.

The energy radiated away by bound-bound transitions constitute almost $99 \%$ of the total radiative losses for $\log _{10}(T) \in$ $\langle 5,6\rangle$. Their contribution decreases to about $87 \%$ for $\log _{10}(T)=$ 7 , to $35 \%$ for $\log _{10}(T)=7.5$ and only to $14.5 \%$ for $\log _{10}(T)=8$.

The elements $\mathrm{C}, \mathrm{N}, \mathrm{O}, \mathrm{Ne}, \mathrm{Mg}, \mathrm{Al}, \mathrm{Si}, \mathrm{S}, \mathrm{Ar}, \mathrm{Ca}, \mathrm{Fe}$, and $\mathrm{Ni}$ produce $\geq 98 \%$ of the bound-bound radiative losses for $\log _{10}(T) \in\langle 5.3,6\rangle$ and more than $98.8 \%$ for $T \in\langle 6,8\rangle$. At $\log _{10}(T)=5$, about $10 \%$ of the bound-bound radiative losses are due to the emission from the He II ions, particularly from the resonance lines at $303.8 \AA$. At $\log _{10}(T)=5.3$, He II accounts only for about $1 \%$ of the bound-bound radiative losses. For $\log _{10}(T) \in\langle 5.3,6\rangle$, other important missing emission lines $(\approx 1 \%)$ are $\mathrm{Na}$ lines. The missing bound-bound radiative losses at $\log _{10}(T) \in\langle 6,8\rangle$ are produced mainly by the elements $\mathrm{Cr}, \mathrm{Na}$, $\mathrm{Mn}, \mathrm{K}$, and Co. Because of the missing contribution from $\mathrm{He}$ and the possible presence of opacity effects for $\log _{10}(T) \lesssim 5$, we compute the bound-bound radiative losses due to these elements in the range of $\log _{10}(T) \in\langle 5,8\rangle$.

Since the majority of the bremsstrahlung emission comes from electron collisions with ionized $\mathrm{H}$ and $\mathrm{He}$, which are missing from the ionization equilibrium calculations for nonthermal distributions, we assume that these elements are completely ionized for $\log _{10}(T) \geq 5.5$ and neglect the contribution from

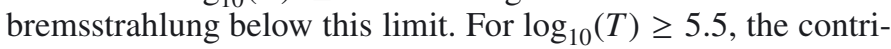
butions from the collisions with ions of the other missing, lowabundance elements are less than $0.3 \%$. The free-free continuum calculations in this paper are thus the most precise. The error is mainly caused by missing contributions from $\mathrm{Na}$ and $\mathrm{Cr}$.

In summary, the combined error produced by the missing free-bound continuum, emission lines and a slight part of the bremsstrahlung, is $\leq 3.5 \%$ for $\log _{10}(T) \in\langle 5.2,7\rangle$ and less than $9 \%$ for $\log _{10}(T) \geq 7$ (Fig. 6). A similar error is expected in computed radiative losses for nonthermal $\kappa$ - and $n$-distributions.

We now proceed to evaluate the bound-bound and free-free diative losses using the approach outlined in Sect. 3 for $\kappa$ - and $n$-distributions. The radiative losses are computed in the logarithmical grid of $T$ with the step of $\Delta \log _{10}(T)=0.02$ dex. For $n$-distributions, $\tau$ is used instead of $T$. We assume the value of $n_{\mathrm{e}}=10^{10} \mathrm{~cm}^{-3}$ and drop the $n_{\mathrm{e}} n_{\mathrm{H}} \approx 8.5 \times 10^{19} \mathrm{~cm}^{-6}$ factor from the computations.

\subsection{Radiative losses for the nonthermal distributions}

The computed bound-bound radiative losses from the 12 elements considered and bremsstrahlung for the $\kappa$-distributions 

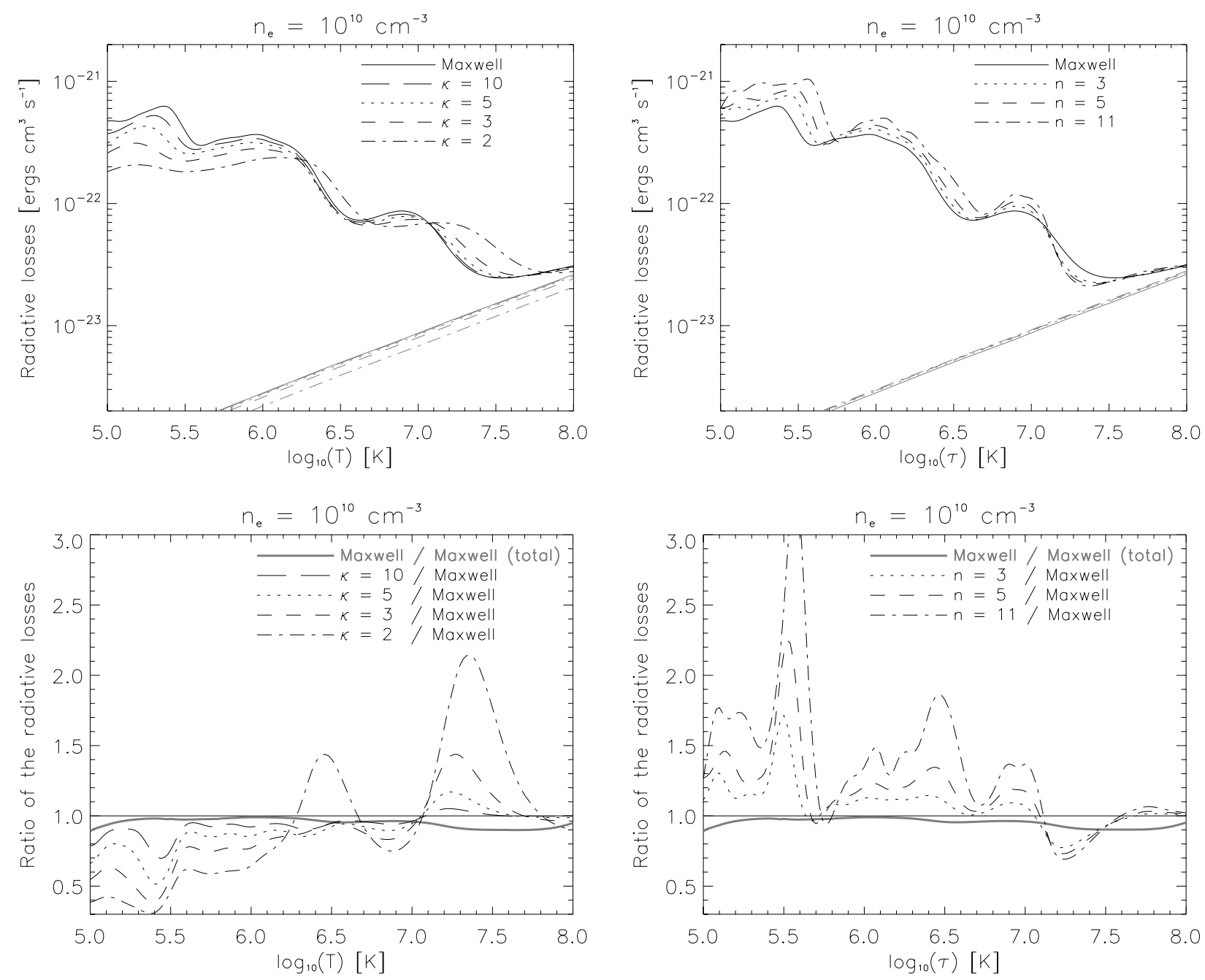

Fig. 6. Radiative-loss functions for the nonthermal distributions and their ratios with respect to the radiative-loss function for Maxwellian distribution. Left column: radiative losses for $\kappa$-distributions; right column: radiative losses for $n$-distributions. Thin gray lines in the top row correspond to the contribution of bremsstrahlung. Thick gray line in the bottom row represents the ratio of the bound-bound and free-free radiative losses to the total radiative losses for Maxwellian distribution.

with $\kappa=2,3,5$, and 10 , and $n$-distributions with $n=3,5$, and 11, together with the Maxwellian distribution are displayed in Fig. 6.

\subsubsection{Radiative losses for the $\kappa$-distributions}

The radiative losses for $\kappa$-distributions are generally smoother and have flatter peaks than for a Maxwellian distribution. The radiative losses are less than radiative losses for Maxwellian distribution for $\log _{10}(T)<7$, with the exception of $\kappa=2$. The ratio of radiative losses for $\kappa$-distributions to the radiative losses for Maxwellian distribution is depicted in the bottom left of the Fig. 6. For $\log _{10}(T)>7$, the radiative losses for $\kappa$-distributions are greater than for Maxwellian distribution. The changes are most pronounced for $\kappa=2$, where the ratio is between 0.3 and 2.2 in the entire interval of $T$. For larger $\kappa$, the ratio is progressively closer to unity.

The peaks and local minima of the radiative-loss functions for $\kappa$-distributions are also shifted with respect to Maxwellian one. The shifts are generally to higher $T$, except for $\log _{10}(T)<$ 5.5 , where the shift is toward lower values of $T$. The location of peaks corresponding to the radiative-loss function for
Table 1. Local minima and maxima of the radiative-loss functions.

\begin{tabular}{lcccccc}
\hline \hline Distribution & \multicolumn{7}{c}{ Local minima location $\left[\log _{10}(T){\left.\text { or } \log _{10}(\tau)\right]}^{\prime}\right.$} \\
\hline$\kappa=2$ & - & - & - & 5.50 & 6.84 & 7.88 \\
$\kappa=3$ & - & - & - & 5.50 & 6.68 & 7.68 \\
$\kappa=5$ & - & - & - & 5.54 & 6.64 & 7.60 \\
$\kappa=10$ & - & - & - & 5.58 & 6.64 & 7.56 \\
\hline Maxwell & - & 5.04 & - & 5.62 & 6.64 & 7.54 \\
\hline$n=3$ & - & 5.18 & - & 5.66 & 6.64 & 7.44 \\
$n=5$ & - & 5.24 & - & 5.70 & 6.66 & 7.40 \\
$n=11$ & 5.14 & 5.36 & 5.46 & 5.78 & 6.72 & 7.38 \\
\hline distribution & local maxima location $\left[\log _{10}(T)\right.$ or $\left.\log _{10}(\tau)\right]$ \\
\hline$\kappa=2$ & - & - & - & 5.20 & 6.12 & 7.14 \\
$\kappa=3$ & - & - & - & 5.18 & 5.96 & 6.98 \\
$\kappa=5$ & - & - & - & 5.26 & 5.94 & 6.92 \\
$\kappa=10$ & - & - & - & 5.30 & 5.94 & 6.90 \\
\hline Maxwell & - & - & - & 5.38 & 5.96 & 6.90 \\
\hline$n=3$ & - & 5.10 & - & 5.46 & 5.98 & 6.90 \\
$n=5$ & - & 5.18 & - & 5.48 & 6.00 & 6.92 \\
$n=11$ & 5.12 & 5.28 & 5.42 & 5.56 & 6.06 & 6.90 \\
\hline
\end{tabular}

Maxwellian distribution are listed in Table 1. The error of the peak or minimum location is equal to the resolution of the curves, i.e., 0.02 dex. 
Contribution to radiative losses from bremsstrahlung (Fig. 6 top left) retains its $T^{1 / 2}$ dependence. The contribution is lower for $\kappa$-distributions than for Maxwellian distribution, although not by much. On average, the contribution is lowered to $98.9 \%$ for $\kappa=10$, to $96.9 \%$ for $\kappa=5$, to $92.2 \%$ for $\kappa=3$, and to $78.4 \%$ for $\kappa=2$.

\subsubsection{Radiative losses for the $n$-distributions}

The radiative losses for $n$-distributions are almost always higher than for a Maxwellian distribution. The notable exception occurs for $\log _{10}(T) \in\langle 7.1,7.6\rangle$, where the radiative losses are up to $30 \%$ smaller than for the Maxwellian distribution. The largest departure of the radiative losses for $n$-distributions occurs at $\log _{10}(\tau)=5.58$ for $n=11$, where the radiative losses for the $n=11$ distribution are 3.4 times more than for the Maxwellian distribution.

The radiative-loss functions for various $n$-distributions are more undulated than those for the Maxwellian distribution. Their peaks are narrower and more numerous than those for the Maxwellian distribution. The locations of peaks and local minima of the radiative-loss functions are also listed in Table 1.

Remarkably, the contribution from bremsstrahlung remains nearly unchanged. For $n=3$, it is $3.4 \%$ higher than for Maxwellian distribution. The increase is $4.8 \%$ for $n=5$ and only $6.3 \%$ for $n=11$. While the $\tau^{1 / 2}$ dependence readily comes from the $T^{1 / 2}$ dependence in Eq. (13) combined with the definition of $\tau$ in Eq. (7), the slowly changing magnitude of the bremsstrahlung contribution with respect to $n$ is the result of the interplay between the decreasing $B_{n}$ factor and the increasing $(y+z)^{(n-1) / 2}$ term in Eq. (13).

The calculated radiative losses for nonthermal distributions are available as two electronic tables at the CDS. The file rloss_k.dat contains the following information. Column 1 lists the value of $\log _{10}(T)$, while Cols. $2-5$ list the radiative losses for the $\kappa$-distributions with $\kappa=2,3,5$, and 10, respectively. The file rloss_n.dat is composed in an analogical manner. In this file, Col. 1 lists the values of $\log _{10}(\tau)$, and Cols. $2-5$ list the radiative losses for $n=1$ (Maxwellian distribution), $n=3,5$, and 11, respectively.

\subsection{Contribution of bound-bound transitions in individual elements}

It is known that for some temperatures the radiative-loss function is dominated by transitions in a single element (e.g., Landi \& Landini 1999; Colgan et al. 2008; Wiersma et al. 2009; Schure et al. 2009). With this in mind, we now investigate the role of bound-bound transitions in individual elements when constituting the radiative-loss function for different distributions. Unlike Colgan et al. (2008), we do not include the contribution of these elements to bremsstrahlung, since bremsstrahlung is dominated by contributions from $\mathrm{H}$ and $\mathrm{He}$.

The contribution of the bound-bound transition from the individual elements are for $\kappa$-distributions depicted in Fig. 7 and for $n$-distributions in Fig. 8. The largest contribution to radiative losses at transition region temperatures comes from oxygen, in particular the $\mathrm{O}_{\mathrm{IV}}, \mathrm{Ov}$, and $\mathrm{O} v \mathrm{vi}$ ions, with the largest contribution coming from the Ov $629.73 \AA$ line. The changes in the contribution function of this line (Fig. 5) are then reflected in the shape of the radiative-loss function, most notably the peak shift (Table 1) toward lesser $T$ for $\kappa$-distributions and larger $\tau$ for $n$-distributions. The total contribution of oxygen to the peak also changes with the type of distribution. It is only $69.1 \%$ for $\kappa=2$, and then rises up to $86.0 \%$ for Maxwellian distribution and up to $88.5 \%$ for $n=11$. The appearance of additional peak at $\log _{10}(\tau)$ between 5.1 and 5.28 for $n$-distributions is caused by the behavior of $\mathrm{C}$, most notably by transitions in $\mathrm{C}_{\text {III }}$ and $\mathrm{C}$ IV ions.

At coronal temperatures, the radiative-loss curve is dominated by contributions from $\mathrm{Fe}$, with maximum contributions ranging from $74.1 \%$ to $82.0 \%$, in increasing order from $\kappa=2$ to $n=11$. For Maxwellian distribution, the maximum contribution is around $77 \%$. According to Figs. 7 and 8, the increase or decrease in radiative losses with the change of the type of the distribution is caused primarily due to the transitions in Fe.

The elements $\mathrm{Ne}, \mathrm{Mg}$, and $\mathrm{Si}$ contribute up to $\approx 20 \%$ to the radiative losses in a given temperature range. The contributions of S, Ca, and Ni approach 5\%. We note that, for a given $T$ or $\tau$, the relative change of the contribution of a given element owing to different distribution with respect to the contribution for Maxwellian distribution can reach a factor 10 or more. This large factor comes from the shift of the ionization peaks to different $T$ or $\tau$.

The contribution of individual elements depends on the assumed elemental abundances, which in this paper are coronal with the FIP enhancement of 3.5 (e.g., Aschwanden 2006, p. 64). The effect of different abundances on the radiative-loss function can be easily obtained by scaling the contribution of given elements in Figs. 7 and 8 by the appropriate constant.

\subsection{Contribution of Fe ions}

Since the radiative losses for coronal temperatures are controlled by $\mathrm{Fe}$, we investigated the contribution of individual $\mathrm{Fe}$ ions, and the results are displayed in Fig. 9. At $\log _{10}(T)>5.6$, the two main peaks of the radiative-loss function are constituted by the ions Fe IX - Fe XV and Fe XVII - Fe XXIII, with the contribution of the Fe IX and Fe XVII locally larger than neigboring ions. This comes from the behavior of the ionization potential, whose derivation has local maxima corresponding to closed-shells, i.e., ions Fe IX and Fe XvII.

The shape of the radiative-loss function peaks again reflects the shape of their constituent ions. In general, the peaks are lower and wider for $\kappa$-distributions, and higher and narrower for $n$ distributions. The location and value of their maxima can change as well. An interesting feature is the change in the Fe xII/Fe XIII radiative output peak ratio with the type of distribution. It is $\approx 1.15$ for Maxwellian distribution, but increases to 1.85 for $n=11$ and decreases rapidly between $\kappa=2$ and 3 to the value of 0.78 for $\kappa=2$. This behavior comes from the combination of the behaviors of both the ionization and excitation equilibria for these ions.

For the $n$-distributions, the contributions from ions Fe XVII Fe XXIII tend to concentrate in a smaller interval of $\tau$, leaving the local minimum of the radiative-loss function constituted almost only by the Fe xxIv. The relatively large distance between Fe xxIV and Fe Xxv peaks are caused by the rapid increase in the ionization potential.

\section{Discussion}

\subsection{Dependence on atomic data}

The latest version of CHIANTI, 6.0, contains an updated ionization equilibrium file, chianti.ioneq (Dere et al. 2009, and references therein). The most notable differences occur for Fe VI, 

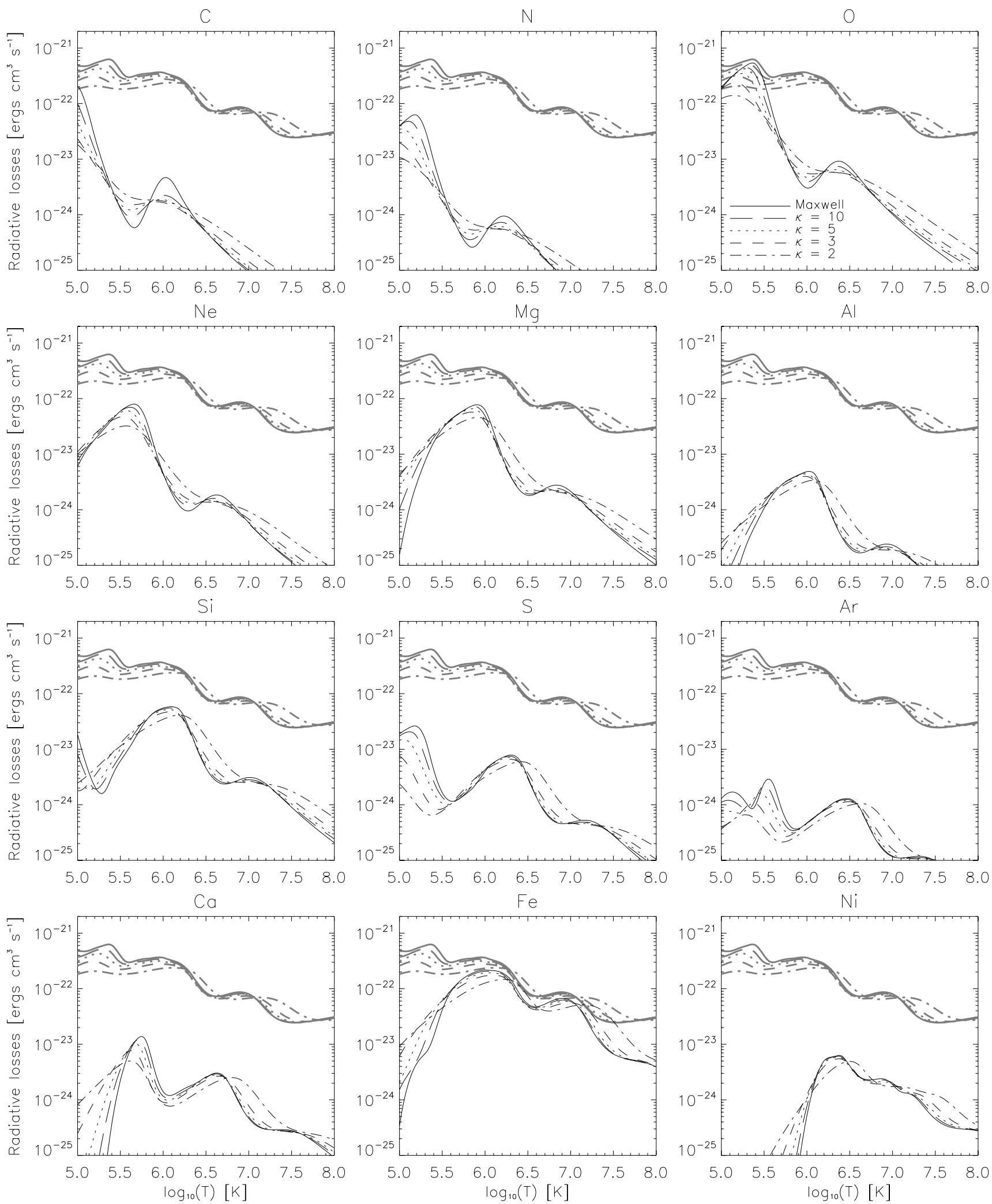

Fig. 7. Contribution to radiative losses for $\kappa$-distributions from the 12 considered elements. Thick gray lines represent the radiative-loss functions computed in Sect. 4.2.

Fe vIII, and Fe Ix. In general, these updated data lead to a different radiative-loss curve. The differences between the radiative loss curve computed with CHIANTI 6.0 using the chianti.ioneq and the curve computed using CHIANTI 5.2 with the mazzotta_etal_ext.ioneq file (Sect. 3.3) are plotted in Fig. 10, and are within $11 \%$. The three main peaks are composed of $\mathrm{O}$ (peak at $\left.\log _{10}(T) \approx 5.45\right)$, Fe and $\mathrm{Si}\left(\right.$ peak at $\left.\log _{10}(T) \approx 6.35\right)$, and $\mathrm{Fe}$ and $\mathrm{Ni}$ (peak at $\log _{10}(T) \approx 7.05$ ).

The ionization and recombination cross-sections used to prepare the ioneq files for nonthermal distributions correspond to 
J. Dudík et al.: The bound-bound and free-free radiative losses for the nonthermal distributions in solar and stellar coronae
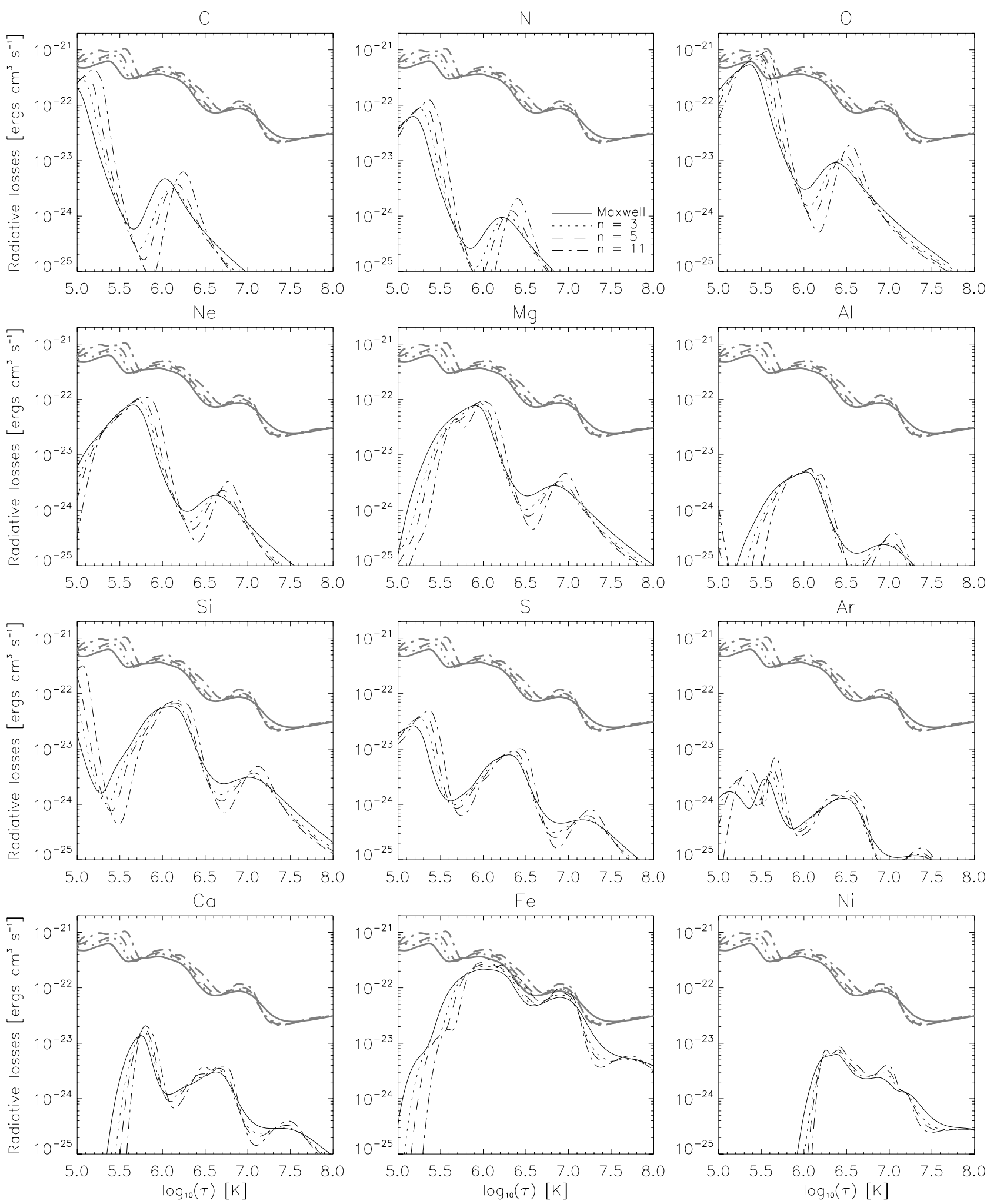

Fig. 8. Same as in Fig. 7, but for $n$-distributions.

the cross-sections used in the calculations of Mazzotta et al. (1998), so the corresponding ionization equilibrium file for Maxwellian distribution must be used if the changes in the radiative-loss curve for nonthermal distribution are to be investigated in a consistent manner. We conclude that the effect of changes in atomic data is within the same order or magnitude as the effect of missing free-bound continuum and is up to several times smaller than the effect of nonthermal distributions (Sect. 4.2).

\subsection{Dependence on $n_{\mathrm{e}}$}

Apart from the $n_{\mathrm{e}} n_{\mathrm{H}}$ factor, the electron density $n_{\mathrm{e}}$ in the coronal approximation only influences the excitation equilibrium. 
A\&A 529, A103 (2011)
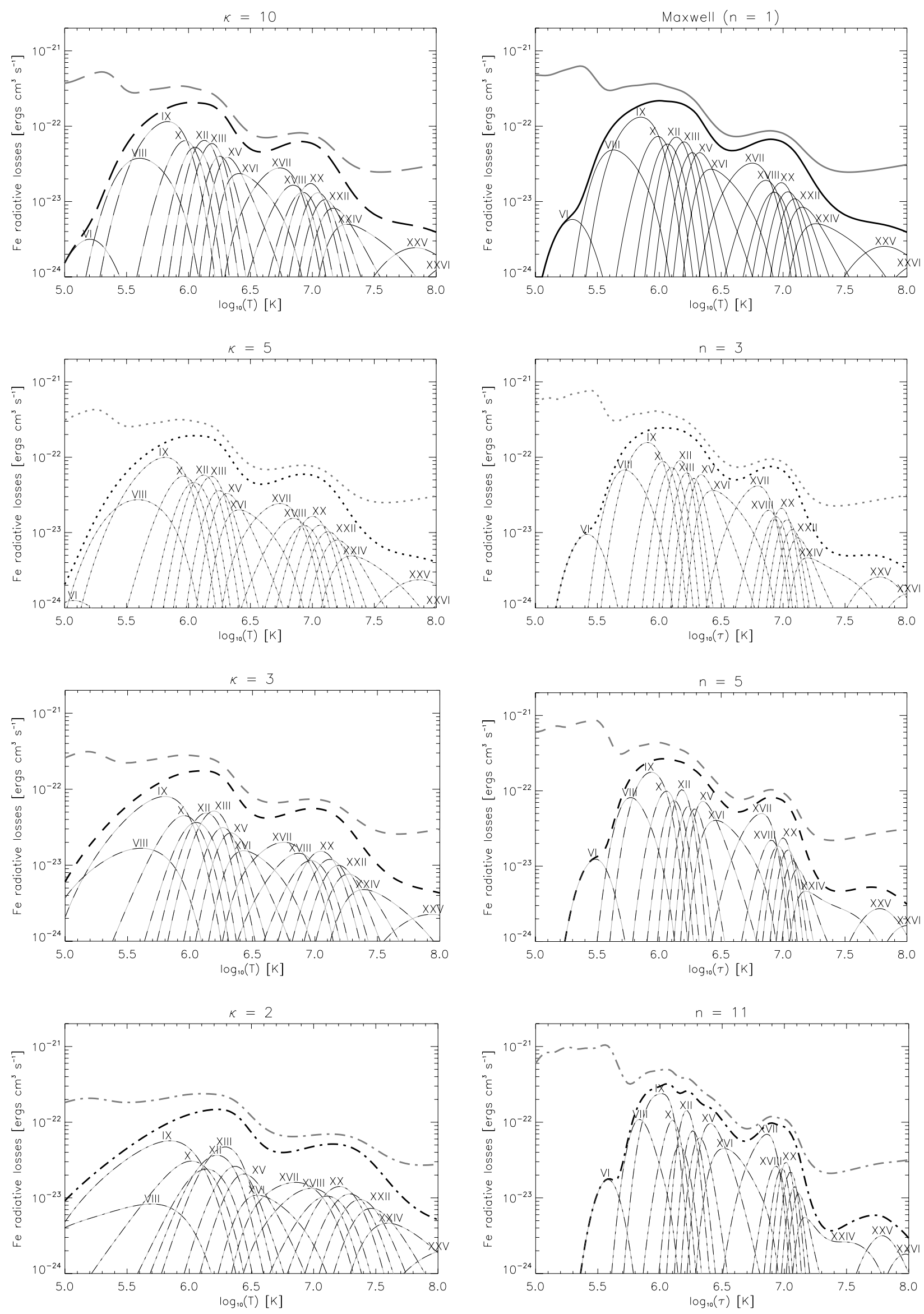

Fig. 9. Contribution of individual Fe ions to the radiative losses for $\alpha$-distributions (left column) and for $n$-distributions (right column). 
J. Dudík et al.: The bound-bound and free-free radiative losses for the nonthermal distributions in solar and stellar coronae

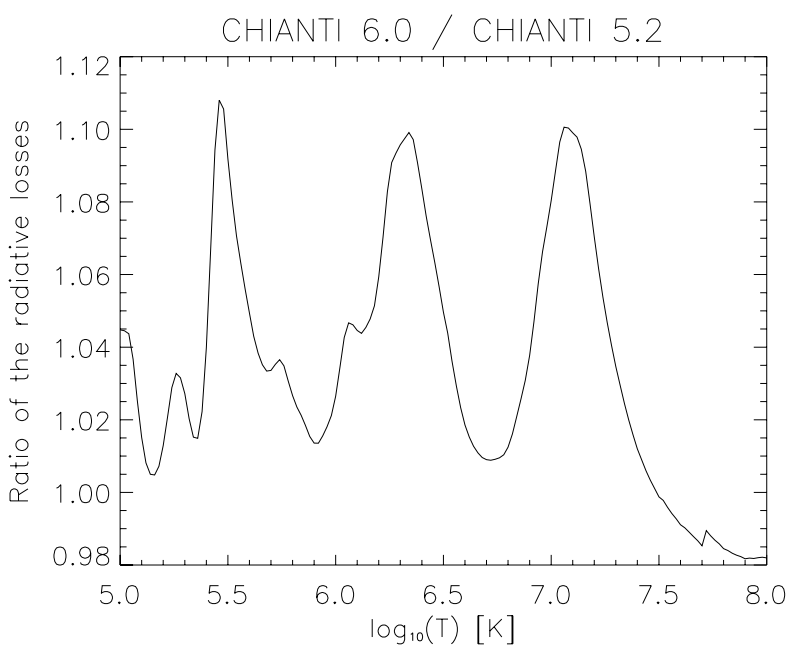

Fig. 10. Ratios of radiative-loss functions computed for Maxwellian distribution with CHIANTI 6.0 to the radiative loss curves computed with CHIANTI 5.2 with the appropriate ionization equilibrium files (see text).
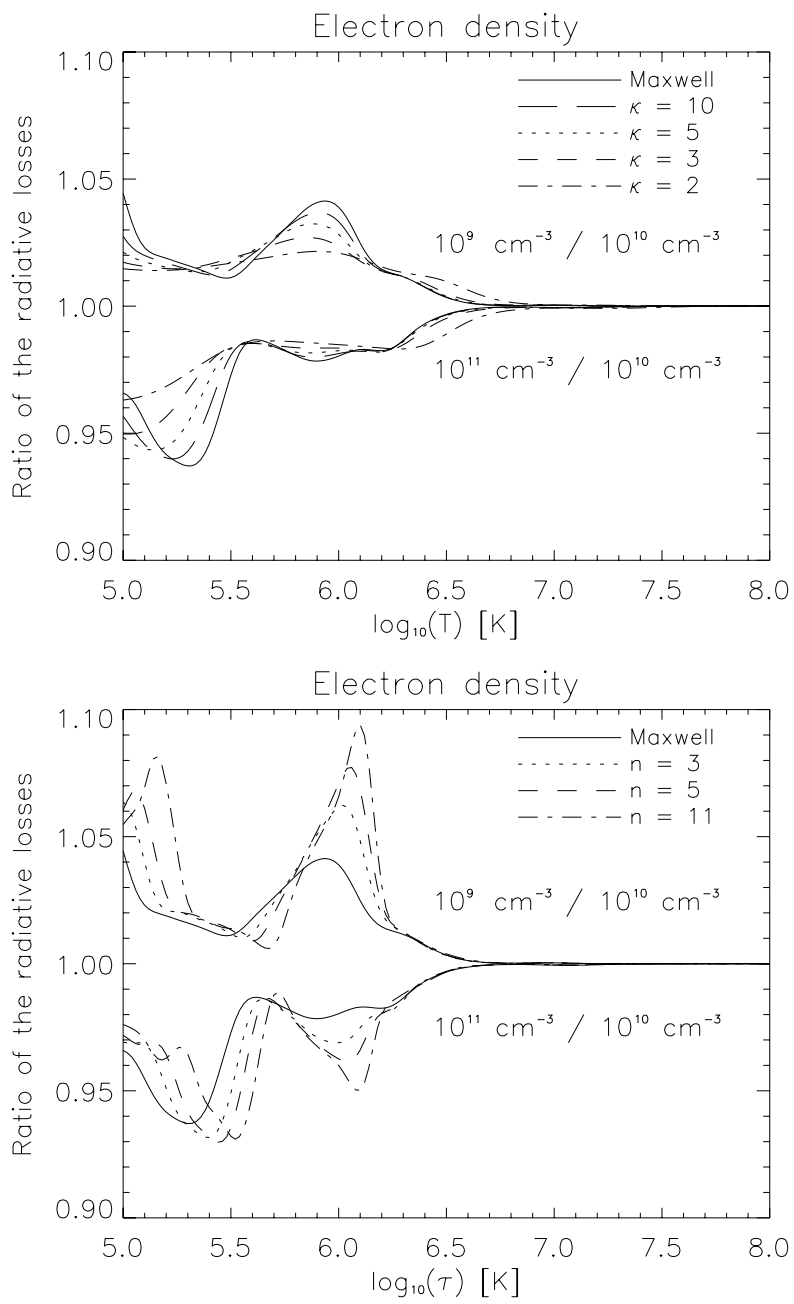

Fig. 11. Ratios of radiative-loss functions computed for $n_{\mathrm{e}}=10^{9}$ and $10^{11} \mathrm{~cm}^{-3}$ to the radiative loss curves for $n_{\mathrm{e}}=10^{10} \mathrm{~cm}^{-3}$ for $\kappa$-distributions (top) and $n$-distributions (bottom).

Figure 11 shows the difference in radiative-loss curves computed for $n_{\mathrm{e}}=10^{9} \mathrm{~cm}^{-3}$ and $10^{11} \mathrm{~cm}^{-3}$ with respect to the radiativeloss curve computed in Sect. 4.2 for $n_{\mathrm{e}}=10^{10} \mathrm{~cm}^{-3}$. For $n_{\mathrm{e}}=$ $10^{9} \mathrm{~cm}^{-3}$, the radiative losses are higher than for $n_{\mathrm{e}}=10^{10} \mathrm{~cm}^{-3}$.
Table 2. Power-law fits to the radiative-loss functions.

\begin{tabular}{lccccc}
\hline \hline Distrib. & $\log _{10}(\chi)$ & $\alpha$ & Distrib. & $\log _{10}(\chi)$ & $\alpha$ \\
\hline$K=10$ & -18.608 & -0.513 & Maxwell & -18.378 & -0.544 \\
$\kappa=5$ & -18.851 & -0.479 & $n=3$ & -18.057 & -0.590 \\
$\kappa=3$ & -19.211 & -0.426 & $n=5$ & -17.872 & -0.614 \\
$\kappa=2$ & -19.782 & -0.340 & $n=11$ & -17.565 & -0.652 \\
\hline
\end{tabular}

The maximum difference is $\lesssim 5 \%$ for $\kappa$-distributions and $\lesssim 10 \%$ for $n$-distributions. For $n_{\mathrm{e}}=10^{11} \mathrm{~cm}^{-3}$, the radiative losses decrease up to $\lesssim 7 \%$ for both $\kappa$-distributions and $n$-distributions.

Between $n_{\mathrm{e}}=10^{9}$ and $10^{10} \mathrm{~cm}^{3}$, the differences for $\log _{10}(T) \leq 5.5$ and $\kappa$-distributions are caused by elements $\mathrm{C}$, $\mathrm{N}, \mathrm{O}$, and $\mathrm{Mg}$. The contribution of $\mathrm{Mg}$ is small for Maxwellian distribution but increases with $\kappa \rightarrow 2$. The peak at $\log _{10}(T) \lesssim 6$ is mainly caused by Fe. For $n_{\mathrm{e}}=10^{11} \mathrm{~cm}^{3}$, the differences are caused mainly by $\mathrm{O}, \mathrm{Si}$, and $\mathrm{Fe}$.

For $n$-distributions, the differences between $n_{\mathrm{e}}=10^{9}$ and $10^{10} \mathrm{~cm}^{3}$ are mainly caused by $\mathrm{C}$ and $\mathrm{Fe}$, with a smaller contribution from $\mathrm{O}$. On the other hand, between $n_{\mathrm{e}}=10^{10}$ and $10^{11} \mathrm{~cm}^{3}$, the changes are dominated by transitions in $\mathrm{O}$ and $\mathrm{Fe}$.

We note that the density-dependent dielectronic recombination processes in Li-like ions (Doyle et al. 2005) were not taken into account during the production of the ioneq files. Similarly, the influence of photoionization (Wiersma et al. 2009) was not taken into account.

\subsection{The effect of photoexcitation}

Photoexcitation can affect transition region line intensities. To investigate its effect on the radiative-loss functions, we included the radiation field with radiation temperature of $6000 \mathrm{~K}$ in the assumed height of $5 \mathrm{Mm}$ above the photosphere. Several examples of the effect of the photoexcitation on the radiative-loss curves are plotted in Fig. 12. The photoexcitation can increase the radiative losses up to $20 \%$. The peak is caused almost entirely by oxygen, with a small contribution from $\mathrm{C}$ and for $n$-distributions $\mathrm{Mg}$ and S.

\subsection{Power-law fits to the radiative losses}

Analytical models of coronal loops frequently utilize power-law fits of the radiative-loss function in the form $\chi T^{\alpha} n_{\mathrm{e}} n_{\mathrm{H}}$ (e.g., Kuin \& Martens 1982; Martens et al. 2000; Dudík et al. 2009b; Martens 2010). The widely used scaling laws of Rosner et al. (1978) and Serio et al. (1981) assume $\alpha=-1 / 2$.

The values of $\chi$ and $\alpha$ obtained by fitting the radiative-loss functions are summarized in Table 2. With decreasing $\kappa$, both $\chi$ and $\alpha$ decrease. With increasing $n$, they both increase. The value of $\alpha=-0.544$ for the Maxwellian distribution is very close to the one used by Rosner et al. (1978) and Serio et al. (1981).

The error of these fits are within $80 \%$ except for $n=$ 11 , where the errors reach $120 \%$. However, for $\log _{10}(T)$ or $\log _{10}(\tau) \in\langle 5.2,7\rangle$, the error is within $50 \%$, so that these fits are suitable for the purposes of analytical models of coronal loops.

Piecewise, continuous power-law fits to the radiative-loss functions are given in Appendix A.

\subsection{Stability of coronal loops}

The absence of thermal conduction may lead to the radiativelydriven thermal instability (e.g., Priest 1982; Aschwanden 2006, p. 277 and 128, respectively), in particular, catastrophic radiative 

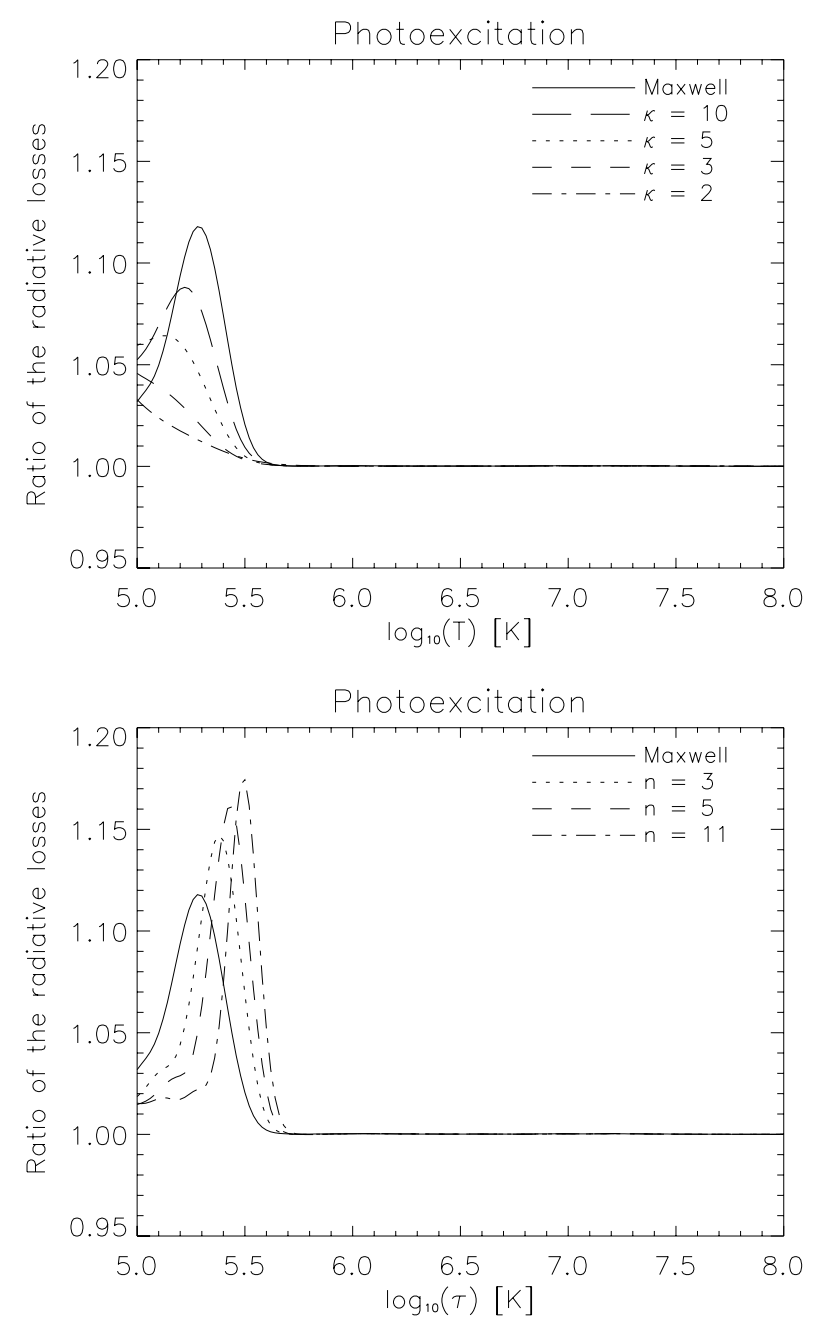

Fig. 12. Ratios of radiative-loss functions computed with and without the effect of photoionization for $\kappa$-distributions (top) and $n$-distributions (bottom).

cooling of the plasma. This happens if the slope $\alpha$ of the radiative-loss function is small enough, so that the time derivative $\partial T / \partial t$ is negative.

This effect may be particularly important for coronal loops, since the extreme-ultraviolet (EUV) loops commonly observed by SOHO/EIT (Delaboudinière et al. 1995), SOHO/CDS (Harrison et al. 1995) or TRACE (Handy et al. 1999) exhibit small a temperature gradient $\mathrm{d} T / \mathrm{d} s \approx 0$ along the loop coordinate $s$ at least for the visible portion of the loop segment (e.g., Aschwanden 2000; Aschwanden et al. 2008; Schmelz et al. 2009). Cooling loops were indeed observed by Winebarger et al. (2003), among others.

We assume that the heating of the coronal plasma takes the following form

$E_{\mathrm{H}}=h n_{\mathrm{e}}^{\beta}$,

where $h$ is a proportionality constant and $\beta$ a parameter. The value of $\beta=0$ corresponds to the constant heating per volume, while the case of $\beta=1$ corresponds to constant heating per particle (Cook et al. 1989). If we assume that the radiative-loss function can be approximated by the form of $E_{\mathrm{R}}=\chi T^{\alpha} n_{\mathrm{e}} n_{\mathrm{H}}$, neglecting thermal conduction then leads to the following criterion for loop stability against the radiatively-driven thermal instability

$\alpha+\beta-2 \geq 0$ or to $\alpha \geq 1$ for $\beta=1$. The reader is referred to, e.g., Aschwanden (2006, p. 129,) for a derivation of this criterion for $\beta=1$.

Survey of the values of $\alpha$ for the radiative-loss functions for nonthermal distributios computed in this paper (Appendix A) yields only one case where $\alpha \geq 1$, for $\log _{10}(\tau) \in\langle 5.66,5.98\rangle$ for the $n$-distribution with $n=11$. We note that this is an extremely nonthermal situation, occurring only intermittently in flares (Kulinová et al. 2011). We thus conclude that if $\beta=1$, stable loops cannot exist without the stabilizing effect of the thermal conduction.

Different outcomes can be obtained if we suppose that the value of $\beta$ is given by equilibrium solutions for coronal loops. According to the scaling laws of Dudík et al. (2009b), valid for $\alpha>-3 / 2, E_{\mathrm{H}}$ is proportional to $n_{\mathrm{e}}^{14 /(7+2 \alpha)}$. The stability condition (18) is then fulfilled if

$\alpha+\frac{14}{7+2 \alpha}-2 \geq 0$

which has two solutions, $\alpha \geq 0$ and $-7 / 2<\alpha \leq-3 / 2$. The latter is not permitted because the scaling laws are only valid for $\alpha>-3 / 2$. There are a number of temperature intervals where $\alpha \geq 0$, i.e., where stable solutions are possible (Tables A.1 and A.2 in Appendix A). The most notable one is $T \gtrsim 3.5 \mathrm{MK}$, corresponding to X-ray loops, which seem to be stable according to observations (e.g., Brooks \& Warren 2009; Warren et al. $2010 \mathrm{~b}$ ). The other stable interval for $T<1 \mathrm{MK}$ may lie outside of the bulk of the differential emission measure (e.g., Brooks et al. 2010; Schmelz et al. 2008, 2009, 2010a,b) and could thus be more difficult to observe.

\section{Conclusions}

We calculated both the radiative losses due to bremsstrahlung and the bound-bound transitions in 12 elements $(\mathrm{C}, \mathrm{N}, \mathrm{O}, \mathrm{Ne}$, $\mathrm{Mg}, \mathrm{Al}, \mathrm{Si}, \mathrm{S}, \mathrm{Ar}, \mathrm{Ca}, \mathrm{Fe}$, and $\mathrm{Ni}$ ) for nonthermal distributions with the assumed value of electron density $n_{\mathrm{e}}=10^{10} \mathrm{~cm}^{-3}$. The 12 elements and bremsstrahlung constitute more than $90 \%$ of the radiative losses for $\log _{10}(T) \in\langle 5,8\rangle$ for the Maxwellian distribution. Changes in atomic data (Sect. 5.1) result in $\leq 11 \%$ changes in the radiative-loss function.

These functions for nonthermal distributions are quite different from the one for Maxwellian distribution. For $\boldsymbol{\kappa}$-distributions, the radiative losses are less than for Maxwellian distributions. One notable exception occurs at $\log _{10}(T)>7$ due to the shift in the contribution from Fe xvII-Fe xxII to higher $T$. Another exception occurs for $\kappa=2$ at $\log _{10}(T) \in\langle 6.2,6.7\rangle$, again because of the behavior of $\mathrm{Fe}$ ions. The peaks in the radiative-loss functions for $\kappa$-distributions are in general flatter and wider than for the Maxwellian distribution. The locations of local minima and maxima may also be shifted.

For $n$-distributions, the radiative losses are generally higher than for Maxwellian distribution. Their peaks are in general narrower and more numerous with the increasing value of parameter $n$, i.e., with the increasing departure from Maxwellian distribution. The peaks and local minima are again shifted.

The contribution from bremsstrahlung increases by only a few percent for $n$-distributions. For $\kappa$-distributions, contribution from bremsstrahlung again decreases by a few percent, except for $\kappa=2$, where the contribution is decreased to $78.4 \%$ with respect to the contribution for Maxwellian distribution.

The radiative-loss functions are slightly sensitive to changes in electron density $n_{\mathrm{e}}$. After dropping the $n_{\mathrm{e}} n_{\mathrm{H}}$ factor, lower $n_{\mathrm{e}}$ means an increase in the radiative losses, and vice versa. The 
J. Dudík et al.: The bound-bound and free-free radiative losses for the nonthermal distributions in solar and stellar coronae

Table A.1. Parametric fit to the radiative-loss functions for the $\kappa$-distributions.

\begin{tabular}{lccccc}
\hline \hline$\kappa=10$ & \multicolumn{5}{c}{$\kappa=5$} \\
\hline $\log _{10}(T)$ & $\log _{10}(\chi)$ & $\alpha$ & $\log _{10}(T)$ & $\log _{10}(\chi)$ & $\alpha$ \\
\hline$\langle 5.00,5.34\rangle$ & -24.223 & +0.557 & $\langle 5.00,5.24\rangle$ & -24.804 & +0.660 \\
$\langle 5.34,5.52\rangle$ & -12.486 & -1.644 & $\langle 5.24,5.50\rangle$ & -16.114 & -0.997 \\
$\langle 5.52,5.94\rangle$ & -22.758 & +0.218 & $\langle 5.50,5.92\rangle$ & -22.980 & +0.250 \\
$\langle 5.94,6.18\rangle$ & -19.325 & -0.360 & $\langle 5.92,6.18\rangle$ & -19.645 & -0.312 \\
$\langle 6.18,6.56\rangle$ & -11.636 & -1.604 & $\langle 6.18,6.56\rangle$ & -11.948 & -1.559 \\
$\langle 6.56,7.00\rangle$ & -23.271 & +0.170 & $\langle 6.56,7.00\rangle$ & -23.276 & +0.168 \\
$\langle 7.00,7.40\rangle$ & -13.118 & -1.282 & $\langle 7.00,7.48\rangle$ & -14.504 & -1.085 \\
$\langle 7.40,8.00\rangle$ & -23.696 & +0.146 & $\langle 7.48,8.00\rangle$ & -23.943 & +0.177 \\
\hline$\kappa=3$ & \multicolumn{5}{c}{$\kappa=2$} \\
\hline $\log _{10}(T)$ & $\log _{10}(\chi)$ & $\alpha$ & $\log _{10}(T)$ & $\log _{10}(\chi)$ & $\alpha$ \\
\hline$\langle 5.00,5.18\rangle$ & -23.656 & +0.416 & $\langle 5.00,5.20\rangle$ & -23.151 & +0.284 \\
$\langle 5.18,5.48\rangle$ & -18.537 & -0.571 & $\langle 5.20,5.52\rangle$ & -20.497 & -0.227 \\
$\langle 5.48,5.94\rangle$ & -23.083 & +0.259 & $\langle 5.52,6.10\rangle$ & -23.009 & +0.229 \\
$\langle 5.94,6.20\rangle$ & -20.391 & -0.195 & $\langle 6.10,6.32\rangle$ & -20.541 & -0.176 \\
$\langle 6.20,6.58\rangle$ & -12.141 & -1.528 & $\langle 6.32,6.70\rangle$ & -12.959 & -1.378 \\
$\langle 6.58,7.04\rangle$ & -23.110 & +0.140 & $\langle 6.70,7.26\rangle$ & -23.276 & +0.199 \\
$\langle 7.04,7.56\rangle$ & -15.443 & -0.946 & $\langle 7.26,7.70\rangle$ & -15.582 & -0.904 \\
$\langle 7.56,8.00\rangle$ & -23.827 & +0.161 & $\langle 7.70,8.00\rangle$ & -21.924 & +0.081 \\
\hline
\end{tabular}

changes are within $10 \%$ for the considered types of distributions. Including the effect of photoexcitation can increase the radiative losses by $20 \%$. This increase is caused by oxygen lines.

The X-ray loops with $6.56 \lesssim \log _{10}(T) \lesssim 7.0$ that satisfy the scaling laws of Dudík et al. (2009b) are stable against the radiatively-driven thermal instability. This stability survives for a wide range of nonthermal distributions.

In short, if there are nonthermal distributions, they will cause significant changes in the radiative-loss function. These changes must be taken into account during modeling of the transition region, flares, and possibly solar corona. The radiative-loss functions for nonthermal distributions computed in this paper are available as electronic files at the CDS.

Acknowledgements. The authors are grateful to the anonymous referee for comments that helped to improve the manuscript. This work was supported by Scientific Grant Agency, VEGA, Slovakia, Grant No. 1/0240/11, Grant IAA300030701 of the Grant Agency of the Academy of Sciences of the Czech Republic, and Grants Nos. 205/09/1705 and 205/09/1469 of the Grant Agency of the Czech Republic. J.D. acknowledges support from Comenius University grants Nos. UK/398/2009 and UK/237/2010. The Solar and Heliospheric Observatory $(\mathrm{SOHO})$ is a project of international cooperation between ESA and NASA. The Transition Region and Coronal Explorer (TRACE) is a mission of the Stanford-Lockheed Institute for Space Research, and part of the NASA Small Explorer program.

\section{Appendix A: Parametric fits to radiative-loss functions}

In this Appendix, we give the parametric fits of the radiative-loss functions of the form $\chi T^{\alpha}$ for Maxwellian and $\kappa$-distributions and in the form of $\chi \tau^{\alpha}$ for $n$-distributions. The values of $\chi$ and $\alpha$ are for the given intervals of $T$ or $\tau$ and a given distribution type indicated in Tables A.1 and A.2. The precision of these fits is $\lesssim 10 \%$ for $\kappa$-distributions and $\lesssim 15 \%$ for $n$-distributions.

We note that these fits are by no means unique. The calculated radiative-loss functions for nonthermal distributions are available at the CDS (see Sect. 4.2), so that the interested reader may produce his/her own fits.
Table A.2. Parametric fit to the radiative-loss functions for the Maxwellian and $n$-distributions.

\begin{tabular}{lccccc}
\hline \hline Maxwellian & \multicolumn{5}{c}{$n=3$} \\
\hline $\log _{10}(\tau)$ & $\log _{10}(\chi)$ & $\alpha$ & $\log _{10}(\tau)$ & $\log _{10}(\chi)$ & $\alpha$ \\
\hline$\langle 5.00,5.40\rangle$ & -23.225 & +0.376 & $\langle 5.00,5.48\rangle$ & -22.918 & +0.331 \\
$\langle 5.40,5.56\rangle$ & -10.914 & -1.906 & $\langle 5.48,5.62\rangle$ & -3.785 & -3.161 \\
$\langle 5.56,5.98\rangle$ & -22.809 & +0.231 & $\langle 5.62,5.96\rangle$ & -24.215 & +0.478 \\
$\langle 5.98,6.20\rangle$ & -18.315 & -0.521 & $\langle 5.96,6.20\rangle$ & -18.101 & -0.548 \\
$\langle 6.20,6.56\rangle$ & -11.268 & -1.657 & $\langle 6.20,6.58\rangle$ & -10.740 & -1.736 \\
$\langle 6.56,6.98\rangle$ & -23.396 & +0.191 & $\langle 6.58,6.98\rangle$ & -24.991 & +0.431 \\
$\langle 6.98,7.40\rangle$ & -12.628 & -1.351 & $\langle 6.98,7.32\rangle$ & -7.955 & -2.013 \\
$\langle 7.40,8.00\rangle$ & -23.788 & +0.158 & $\langle 7.32,8.00\rangle$ & -24.577 & +0.259 \\
\hline$n=5$ & \multicolumn{5}{c}{$n=11$} \\
\hline $\log _{10}(\tau)$ & $\log _{10}(\chi)$ & $\alpha$ & $\log _{10}(\tau)$ & $\log _{10}(\chi)$ & $\alpha$ \\
\hline$\langle 5.00,5.50\rangle$ & -22.786 & +0.314 & $\langle 5.00,5.58\rangle$ & -22.466 & +0.268 \\
$\langle 5.50,5.66\rangle$ & -5.456 & -2.833 & $\langle 5.58,5.74\rangle$ & -3.269 & -3.173 \\
$\langle 5.66,5.98\rangle$ & -24.635 & +0.551 & $\langle 5.74,5.90\rangle$ & -28.263 & +1.174 \\
$\langle 5.98,6.24\rangle$ & -17.532 & -0.635 & $\langle 5.90,6.10\rangle$ & -23.028 & +0.286 \\
$\langle 6.24,6.62\rangle$ & -10.627 & -1.741 & $\langle 6.10,6.70\rangle$ & -13.278 & -1.314 \\
$\langle 6.62,6.96\rangle$ & -25.812 & +0.554 & $\langle 6.70,6.96\rangle$ & -26.610 & +0.676 \\
$\langle 6.96,7.32\rangle$ & -7.842 & -2.028 & $\langle 6.96,7.30\rangle$ & -5.083 & -2.414 \\
$\langle 7.32,8.00\rangle$ & -24.740 & +0.280 & $\langle 7.30,8.00\rangle$ & -24.740 & +0.280 \\
\hline
\end{tabular}

\section{References}

Anderson, S. W., Raymond, J. C., \& van Ballegooijen, A. 1996, ApJ, 457, 939 Aschwanden, M. J. 2006, Physics of the Solar Corona: An Introduction (Chichester, United Kingdom: Praxis Publishing Ltd.)

Aschwanden, M. J., Nightingale, R., Alexander, D., et al. 2000, ApJ, 541, 1059 Aschwanden, M. J., Nitta, N. V., Wuelser, J.-P., \& Lemen J. R. 2008, ApJ, 680, 1477

Brooks, D. H, \& Warren, H. P. 2008, ApJ, 687, 1363

Brooks, D. H., \& Warren, H. P. 2009, ApJ, 703, L10

Brooks, D. H., Warren, H. P., Williams, D. R., \& Watanabe, T. 2010, ApJ, 705, 1522

Colgan, J, Abdallah, J. Jr., Sherrill, M. E., et al. 2009, ApJ, 689, 585

Collier, M. R. 2004, Adv. Space Res., 33, 2108

Collier, M. R., Hamilton, D. C., Gloeckler, G., Bochsler, P., \& Sheldon, R. B. 1996, Geophys. Res. Lett., 23, 1191

Cook, J. W., Cheng, C.-C., Jacobs, V. L., \& Antiochos, S. K. 1989, ApJ, 338, 1176

Culhane, J. L., Harra, L. K., James, A. M., et al. 2007, Sol. Phys., 243, 19

Delaboudinière, J.-P., Artzner, G. E., Brunaud, J., et al. 1995, Sol. Phys., 162, 291

Dere, K. P, Landi, E., Mason, H. E., Monsignori Fossi, B. C., \& Young, P. R. 1997, A\&AS, 125, 149

Dere, K. P., Landi, E., Young, P., et al. 2009, A\&A, 498, 915

Doyle, J. G., Summers, H. P., \& Bryans, P. 2005, A\&A, 430, L29

Dudík, J., Kulinová, A., Dzifčáková, E., \& Karlický, M. 2009a, A\&A, 505, 1255

Dudík, J., Dzif̌cáková, E., Karlický, M., \& Kulinová, A. 2009b, A\&A, 502, 957

Dufton, P. L., Kingston, A. E., \& Keenan, F. P., 1984, ApJ, 280, L35

Dzif̌cáková, E. 1992, Sol. Phys., 140, 247

Dzifčáková, E. 1998, Sol. Phys., 187, 317

Dzifčáková, E. 2000, Sol. Phys., 196, 113

Dzif̌cáková, E. 2002, Sol. Phys., 208, 91

Dzifčáková, E. 2006a, Sol. Phys., 234, 243

Dzifčáková E. 2006b, in Proc. SOHO-17, 10 Years of SOHO and Beyond, ed. H. Lacoste, \& L. Ouwehand, ESA SP-617, 89.1

Dzifčáková, E., \& Karlický, M. 2008, Sol. Phys., 250, 329

Dzifčáková, E., \& Kulinová, A. 2001, Sol. Phys., 203, 53

Dzifčáková, E., \& Kulinová, A. 2003, Sol. Phys., 218, 41

Dzifčáková, E., \& Kulinová, A. 2010, Sol. Phys., 263, 25

Dzifčáková, E., \& Kulinová, A. 2011, A\&A, submitted

Dzifčáková, E., \& Tóthová, D. 2007, Sol. Phys., 240, 211

Dzifčáková, E., Kulinova, A., Chifor, C., et al. 2008, A\&A, 488, 311

Feldman, U., Mandelbaum, P., Seely, J. L., Doschek, G. A., \& Gursky, H. 1992, ApJS, 81, 387

Golub, L., \& Pasachoff, J. M. 1997, The solar corona (Cambridge, United Kingdom: Cambridge University Press)

Grevesse, N., \& Sauval, A. J. 1998, Space Sci. Rev., 85, 161 
Gudiksen, B. V., \& Nordlund, Å. 2005a, ApJ, 618, 1020

Gudiksen, B. V., \& Nordlund, A.. 2005b, ApJ, 618, 1031

Handy, B. N., Acton, L. W., Kankelborg, C. C., et al. 1999, Sol. Phys., 187, 229

Harrison, R. A., Sawyer, E. C., Carter, M. K., et al. 1995, Sol. Phys., 162, 233

Kašparová, J., \& Karlický, M. 2009, A\&A, 497, L13

Klimchuk, J. A. 2006, Sol. Phys., 234, 41

Klimchuk, J. A., Patsourakos, S., \& Cargill, P. J. 2008, ApJ, 682, 1351

Klimchuk, J. A., Karpen, J. T., \& Antiochos, S. K. 2010, ApJ, 714, 1239

Kosugi, T., Matsuzaki, K., Sakao, T., et al. 2007, Sol. Phys., 234, 3

Kuin, N. P. M., \& Martens, P. C. H. 1982, A\&A, 108, L1

Kulinová, A., Kasparová, J., Dzifčáková, E., et al. 2011, in 16th Cambridge

workshop on cool stars, stellar systems and the sun, ed. C. Johns-Krull, ASP

Conf. Ser., submitted

Landini, M., \& Monsignori Fossi, B. C. 1991, A\&AS, 91, 183

Landi, E., \& Landini, M. 1999, A\&A, 347, 401

Landi, E., Feldman, U., \& Dere, K. P. 2002, ApJ, 139, 281

Landi, E., Del Zanna, G., Dere, K. P., Mason, H. E., \& Landini, M. 2006, ApJS, 162,261

Leubner, M. P. 2004, ApJ, 604, 469

Leubner, M. P. 2005, ApJ, 632, L1

Leubner, M. P. 2008, Nonlinear Proc. Geophys., 15, 531

Ljepojevic, N. N., \& MacNiece, P. 1987, Sol. Phys., 117, 123

Maksimovic, M., Pierrard, V., \& Lemaire, J. F. 1997, A\&A, 324, 725

Martens, P. C. H. 2010, ApJ, 714, 1290

Martens, P. C. H., Kankelborg, C. C., \& Berger, T. E. 2000, ApJ, 537, 471

Mazzotta, P., Mazzitelli, G., Colafrancesco, S., \& Vittorio, N. 1998, A\&AS, 133, 403

McWhirter, R. W. P., Thonemann, P. C., \& Wilson, R. 1975, A\&A, 40, 63

Müller, D. A. N., Hansteen, V. H., \& Peter, H. 2003, A\&A, 441, 605

Owocki, S. P., \& Scudder, J. D. 1983, ApJ, 270, 758

Phillips, K. J. H., Feldman, U., \& Landi E. 2008, Ultraviolet and X-ray Spectroscopy of the Solar Atmosphere (Cambridge, United Kingdom: Cambridge University Press)

Pierrard, V., \& Lazar, M. 2010, Sol. Phys., 267, 153

Pinfield, D. J., Keenan, F. P., Mathioudakis, M., et al. 1999, ApJ, 527, 1000

Priest, E. R. 1982, Solar Magnetohydrodynamics (Dordrecht, Holland: D. Reidel

Publishing Company)

Rhee, T., Chang-Mo, R., \& Yoon, P.-H. 2006, J. Geophys. Res., 111, A09107
Rosner, R., Tucker, W. H., \& Vaiana, G. S. 1978, ApJ, 220, 643

Roussel-Dupre, R. 1980, Sol. Phys., 68, 265

Schmelz, J. T., Scott, J. T., \& Rightmire, L. A. 2008, ApJ, 684, L115

Schmelz, J. T., Nasraoui, K., Rightmire, L. A., et al. 2009, ApJ, 691, 503

Schmelz, J. T., Kashyap, V. L., Saar, A. H., et al. 2010a, ApJ, 704, 863

Schmelz, J. T., Kimble, J. A., Jenkins, B. S., et al., 2010b, ApJ, 725, L34

Schrijver, C. J., Sandman, A. W., Aschwanden, M. J., \& DeRosa, M. L. 2004, ApJ, 615, 512

Schure, K. M., Kosenko, D., Kaastra, J. S., Keppens, R., \& Vink, R. 2009, A\&A, 508,751

Scudder, J. D. 1992, ApJ, 398, 319

Scudder, J. D., \& Olbert, S. 1979a, J. Geophys. Res., 84, 2755

Scudder, J. D., \& Olbert, S. 1979b, J. Geophys. Res., 84, 6603

Seely, J. F., Feldman, U., \& Doschek, G. A. 1987, ApJ, 319, 541

Serio, S., Peres, G., Vaiana, G. S., et al. 1981, ApJ, 243, 288

Shoub, E. C. 1983, ApJ, 266, 339

Sutherland, R. S. 1998, MNRAS, 300, 321

Sylwester, J., Gaicki, I., Kordylewski, Z., et al. 2005, Sol. Phys., 226, 45

Tandberg-Hanssen, E., \& Emslie, A. G. 1988, The Physics of Solar Flares (Cambridge, United Kingdom: Cambridge University Press)

Tucker, W. H., \& Koren, M. 1971, ApJ, 168, 283

Vasyliunas, V. M. 1968, in Proc. Physics of the Magnetosphere, ed. R. L. D. Carrovilano, \& J. F. McClay, ASSL, 10, 622

Vocks, C., \& Mann, G. 2003, ApJ, 593, 1134

Vocks, C., Mann, G., \& Rausche, G. 2008, A\&A, 480, 527

Wannawichian, S. S., Ruffolo, D., \& Kartawykh, Yu. Yu. 2003, ApJS, 146, 443

Warren, H. P., \& Winebarger, A. R. 2006, ApJ, 645, 711

Warren, H. P., \& Winebarger, A. R. 2007, ApJ, 666, 1245

Warren, H. P., Kim, D. M., DeGiorgi, A. M., \& Ugarte-Urra, I. 2010a, ApJ, 713, 1095

Warren, H. P., Winebarger, A. R., \& Brooks, D. H. 2010b, ApJ, 711, 228

Wiersma, R. C. P., Schaye, J., \& Smith, B. D. 2009, MNRAS, 393, 99

Wilhelm, K., Curdt, W., Marsch, E., et al. 1995, Sol. Phys., 162, 189

Winebarger, A. R., Warren, H. P., \& Seaton, D. B. 2003, ApJ, 593, 1164

Yoon, P. H., Rhee, T., \& Chang-Mo, R. 2006, J. Geophys. Res., 111, A09106

Zhitnik, I. A., Kuzin, S. V., Urnov, A. M., et al. 2005, Astron. Lett. 31, 37

Zouganelis, I. 2008, J. Geophys. Res., 113, A08111 